Check for updates

Cite this: Chem. Sci., 2019, 10, 1023

๑ All publication charges for this article have been paid for by the Royal Society of Chemistry

Received 25th September 2018 Accepted 22nd October 2018

DOI: $10.1039 / \mathrm{c} 8 \mathrm{sc} 04255 a$

rsc.li/chemical-science

\section{A highly soluble, crystalline covalent organic framework compatible with device implementation $\uparrow$}

\author{
Lingling Wang, (D) ${ }^{a}$ Cheng Zeng, ${ }^{a}$ Hong $X u^{b}$ Panchao Yin, (D) ${ }^{\text {ac }}$ Dongcheng Chen, ${ }^{a}$ \\ Jian Deng, (D) ${ }^{a} \mathrm{Mu} \mathrm{Li},{ }^{\text {ac }}$ Nan Zheng, ${ }^{a}$ Cheng Gu (D) *a and Yuguang Ma (D) *a
}

Covalent organic frameworks (COFs) have emerged as a tailor-made platform for designing nextgeneration two-dimensional materials. However, COFs are produced as insoluble and unprocessable solids, which precludes the preparation of thin films for optoelectronic applications. Here, we report designed synthesis of a highly soluble yet crystalline COF material through the regulation of its interlayer interactions. The resulting COF is remarkably soluble in a variety of organic solvents and forms stable true solutions with retention of its layered structure. These unique features endow the COF with solution processability; high-quality, large-area COF films can be produced on various substrates in a high-throughput and efficient manner, with good control over the film thickness, making this material compatible with a variety of device applications. The films are electrically anisotropic; the intra-layer carrier conduction is inhibited, while the inter-layer carrier migration is outstanding, showing the highest conductivity among all reported COF materials. Our highly soluble and processable COF may open new pathways for realising high-performance COF-based optoelectronic devices with diverse functions.

\section{Introduction}

Covalent organic frameworks (COFs) are a new class of crystalline porous materials that allow the atomically precise integration of desirable $\pi$ units to create predesigned skeletons and nanopores, and their unique features of well-defined crystalline porous structure, high stability together with tailored functionalities make them promising materials for many applications. ${ }^{\mathbf{1 - 4}}$ Recently, significant progress has been made in the structural diversity and complexity of COFs for functional exploration. ${ }^{5-11}$ Nevertheless, a large number of challenges remain in the COF field including their poor processability. COFs are inherently crosslinked and are produced as insoluble and unprocessable powders. The limited utility of this form prevents the use of COFs in many applications. For example, two-dimensional (2D) COFs are ideally suited for electronic devices by virtue of their well-defined lattice with $\pi$ functionality. ${ }^{12,13}$ However, they are difficult to form highquality thin films, and cannot be reliably interfaced onto electrodes. Thus COF materials-based optoelectronic devices usually

${ }^{a}$ State Key Laboratory of Luminescent Materials and Devices, Institute of Polymer Optoelectronic Materials and Devices, South China University of Technology, Guangzhou 510640, P. R. China. E-mail: gucheng@scut.edu.cn; ygma@scut.edu.cn ${ }^{b}$ Institute of Nuclear and New Energy Technology, Tsinghua University, Beijing 100084, P. R. China

'South China Advanced Institute for Soft Matter Science and Technology, South China University of Technology, Guangzhou 510640, P. R. China

$\dagger$ Electronic supplementary information (ESI) available. See DOI: $10.1039 / \mathrm{c} 8 \operatorname{sc} 04255 \mathrm{a}$ exhibit inferior performance. Monolayer investigation and film preparation are highlighted as bottleneck issues of "critical importance" in recent reviews of the COF field. ${ }^{1}$ Currently, there are several approaches to prepare COF nanosheets or films. A typically used method is substrate-assisted deposition, ${ }^{\mathbf{1 4 , 1 5}}$ in which film-like COFs are deposited onto certain substrates during their organic synthesis. Nevertheless, this method is not a highthroughput one as the majority of COFs remain as powders in solutions. A few physical approaches, such as ultrasonication, ${ }^{\mathbf{1 6}}$ ball milling, ${ }^{17,18}$ and mechanical delamination, ${ }^{19,20}$ have been utilised to exfoliate COF powders into nanosheets. However, these physical methods usually break the COFs into small pieces, decreasing the quality of the materials. A facile synthetic method for the preparation of COF films is interfacial polymerisation in which the condensation reaction occurs at the liquid-liquid interface, ${ }^{21,22}$ allowing the formation of film-like COF materials; unfortunately, this method is difficult to scale up. A newly developed method to prepare colloidal COF suspensions is to add acetonitrile as a stabilizer. ${ }^{23,24}$ However, this strategy was only appropriate for boronate ester-linked COFs, which were less useful for optoelectronic devices due to the stability issues of these materials. Recently, a bulky synthesis of ionic COFs was developed via the incorporation of cationic or anionic species into the frameworks. ${ }^{25-28}$ Several ionic COFs could be exfoliated into nanosheets upon mixing with solvents, ${ }^{25,26}$ but these COFs are not really soluble in solvents and show rather limited solubility and small sizes of the exfoliated nanosheets; the ionic COF suspensions could not be employed for film preparation by solution- 
processing methods. The development of a methodology for the direct synthesis of solution-processable COFs remains a substantial challenge but is of great importance for the further advancement of this field..$^{-4}$

Herein, we report a highly soluble yet crystalline COF material by encoding high-density electrostatic repulsion into the COF skeleton to withstand the inter-layer $\pi-\pi$ stacking. The resulting COF features obvious crystallinity, while it could readily dissolve in various organic solvents to form true solutions and self-exfoliate into large-area monolayer or multilayer nanosheets. Importantly, the high solubility of this COF allows us to simply prepare high-quality COF films, which allows the investigation of its intra-layer and inter-layer properties, such as its electronic properties. In this work, we reveal for the first time the high, yet anisotropic, conductivity of the COF films.

\section{Results and discussion}

We chose two building blocks featuring opposing properties affecting the crystallinity of the COFs. The knot monomer $4,4^{\prime}, 4^{\prime \prime}, 4^{\prime \prime \prime}$-(pyrene-1,3,6,8-tetrayl)tetraaniline (Py) exhibits strong $\pi-\pi$ interactions and tends to form crystalline stacks, while the linker monomer 1,1-bis(4-formylphenyl)-4,4'-bipyridinium dichloride $\left(\mathrm{Vg}^{2+} \cdot 2 \mathrm{Cl}^{-}\right)$, with its inherently high charge density, provides strong electrostatic repulsion and hampers the stacking of COFs. By integrating both of these factors into the COF skeleton, the PyVg-COF (Fig. 1a) was synthesised via reticular polycondensation of Py with $\mathrm{Vg}^{2+} \cdot 2 \mathrm{Cl}^{-}$under solvothermal conditions. The optimal reaction conditions for the synthesis of the PyVgCOF were $N, N$-dimethylacetamide (DMAc)/mesitylene/6 M AcOH (1/ $9 / 1 \mathrm{v} / \mathrm{v}$ ) at $120^{\circ} \mathrm{C}$ for 7 days, and the desired product was obtained as a brownish-red solid in a $38 \%$ isolated yield. The PyVg-COF was unambiguously characterised by various analytical methods (Fig. S1-S5 and Table S1†).

The crystal structure of the PyVg-COF was determined by using synchrotron powder X-ray diffraction (PXRD) measurements in conjunction with structural simulations. The synchrotron PXRD pattern of the PyVg-COF revealed an obvious peak at $2 \theta=2.54^{\circ}$ and several small peaks centred at $2 \theta=1.55^{\circ}$, $1.95^{\circ}, 4.99^{\circ}, 7.20^{\circ}, 8.07^{\circ}$ and $14.34^{\circ}$ (Fig. 2). To determine the optimal crystal structures, crystal models were generated based on the geometry of the building blocks. The optimal cell dimensions were $a=56.16 \AA$, $b=47.87 \AA$, $c=6.48 \AA$, and $a=\beta$ $=\gamma=90^{\circ}$ (Table S $2 \dagger$ ). Using the structures of the monolayers, two stacked configurations, that is, eclipsed AA and staggered $\mathrm{AB}$ modes, were generated and optimised (Fig. 1b and $\mathrm{c}$ and Tables S3 and S4 $\dagger$ ). The simulated PXRD pattern of the staggered $\mathrm{AB}$ model was in good agreement with the experimental pattern and all the peaks in the experimental pattern could be well indexed, which confirmed that the PyVg-COF adopted a staggered $\mathrm{AB}$ stacking arrangement.

The porosity of the PyVg-COF was characterised by $\mathrm{N}_{2}$ and $\mathrm{CO}_{2}$ adsorption isotherms (Fig. S6a $\dagger$ ) from which the BrunauerEmmett-Teller (BET) surface area of the PyVg-COF was

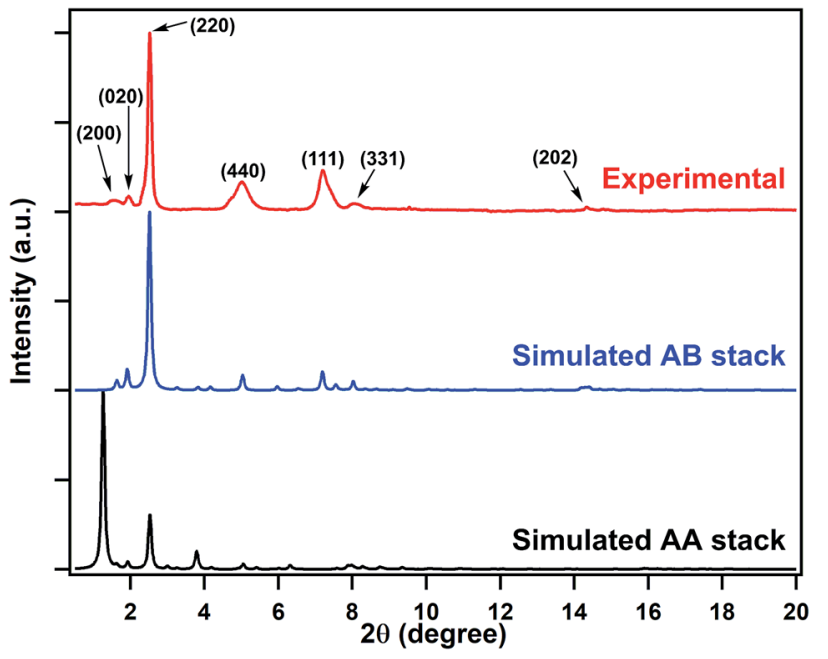

Fig. 2 Synchrotron PXRD patterns of the PyVg-COF: experimentally observed (red curve) and the patterns simulated based on the eclipsed AA (black curve) and staggered AB (blue curve) stacking modes.
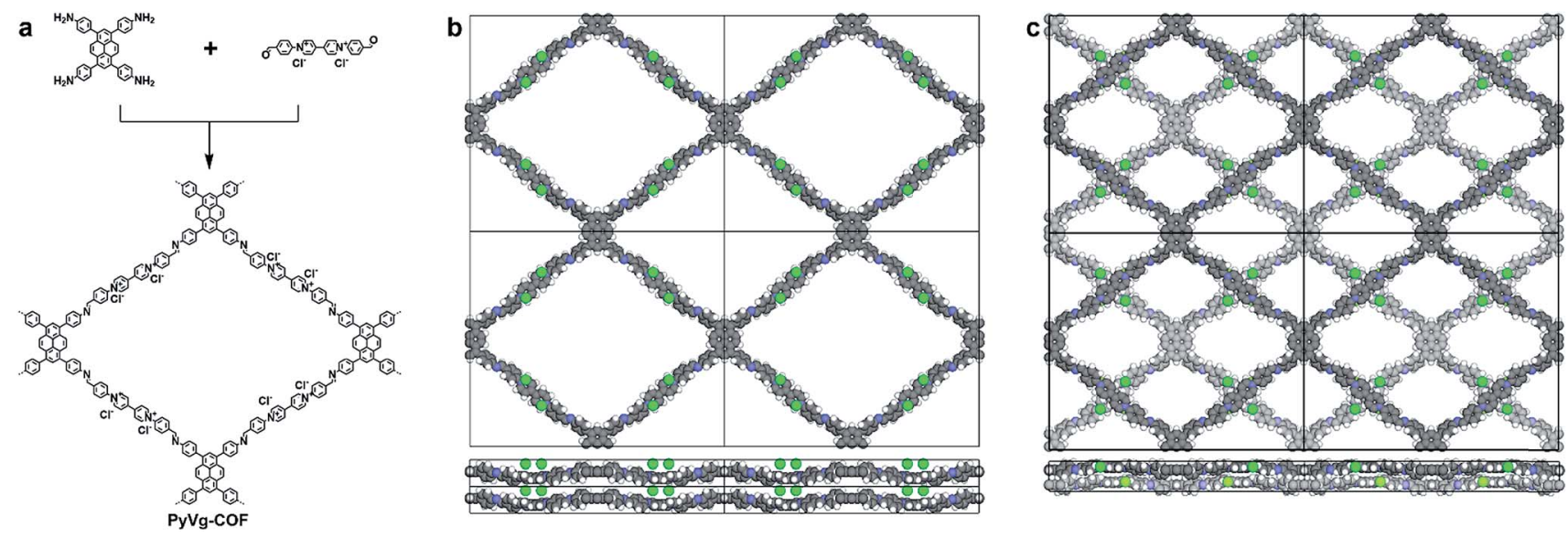

Fig. 1 (a) Schematic representation of the synthesis of the PyVg-COF. (b) Top and side views of the PyVg-COF in the eclipsed AA stacking mode (C: grey; N: blue; Cl: green; H: white). (c) Top and side views of the PyVg-COF in the staggered AB stacking mode (dark grey and light grey show the two staggered layers). 
calculated to be $348 \mathrm{~m}^{2} \mathrm{~g}^{-1}$ from the $\mathrm{CO}_{2}$ adsorption isotherm curve at $273.15 \mathrm{~K}$, and the pore sizes were determined to be 1.20 and $1.56 \mathrm{~nm}$, respectively (Fig. S6b $\dagger$ ). The pore size distribution corresponded well with the two pore sizes generated by $\mathrm{AB}$ stacking. These results demonstrated that the PyVg-COF is highly porous with a large surface area. Surprisingly, while the PyVg-COF adsorbed very little $\mathrm{N}_{2}$ at $77.35 \mathrm{~K}$ and $273.15 \mathrm{~K}$, it adsorbed a substantial amount of $\mathrm{CO}_{2}\left(196 \mathrm{mg} \mathrm{g}^{-1}\right)$ at $273.15 \mathrm{~K}$ (Fig. S6a $\dagger$ ), which is among the highest performances of COFs reported to date, ${ }^{29-31}$ and is also comparable to those of other top-class members. ${ }^{32-34}$ The $\mathrm{CO}_{2} / \mathrm{N}_{2}$ selectivity calculated from the ideal absorbed solution theory (IAST) reached as high as 96 at a relative pressure of 0.2 (Fig. S6c $\dagger$ ). In the PyVg-COF, the staggered structure reduced the pore size, and the strong polarity of the pores caused them to selectively adsorb $\mathrm{CO}_{2}$ and exclude nonpolar $\mathrm{N}_{2}$. At 298.15 K, the PyVg-COF adsorbed less $\mathrm{CO}_{2}\left(134 \mathrm{mg} \mathrm{g}^{-1}\right)$. To clarify the nature of $\mathrm{CO}_{2}$ adsorption, the isosteric heat of adsorption $\left(Q_{\mathrm{st}}\right)$ was calculated using the Clausius-Clapeyron equation from the $\mathrm{CO}_{2}$ adsorption isotherms measured at 273.15 and $298.15 \mathrm{~K}$ (Fig. S6d $\dagger$ ). The $Q_{\text {st }}$ value was as high as $36.1 \mathrm{~kJ} \mathrm{~mol}^{-1}$, which further confirmed the strong interactions between the framework and $\mathrm{CO}_{2}$. Thus, by virtue of its highly polar pores, which form strong interactions with polar gas molecules, the PyVg-COF adsorbs a significant amount of $\mathrm{CO}_{2}$ and a negligible amount of $\mathrm{N}_{2}$, making it an outstanding $\mathrm{CO}_{2}$ adsorbent.

Remarkably, by simple manual shaking, the PyVg-COF readily dissolves in various organic solvents (ESI Video $\dagger$ ), such as $N$-methyl pyrrolidone (NMP), dimethyl sulfoxide (DMSO), $N, N$-dimethylformamide (DMF), $N, N$-diethylformamide (DEF),
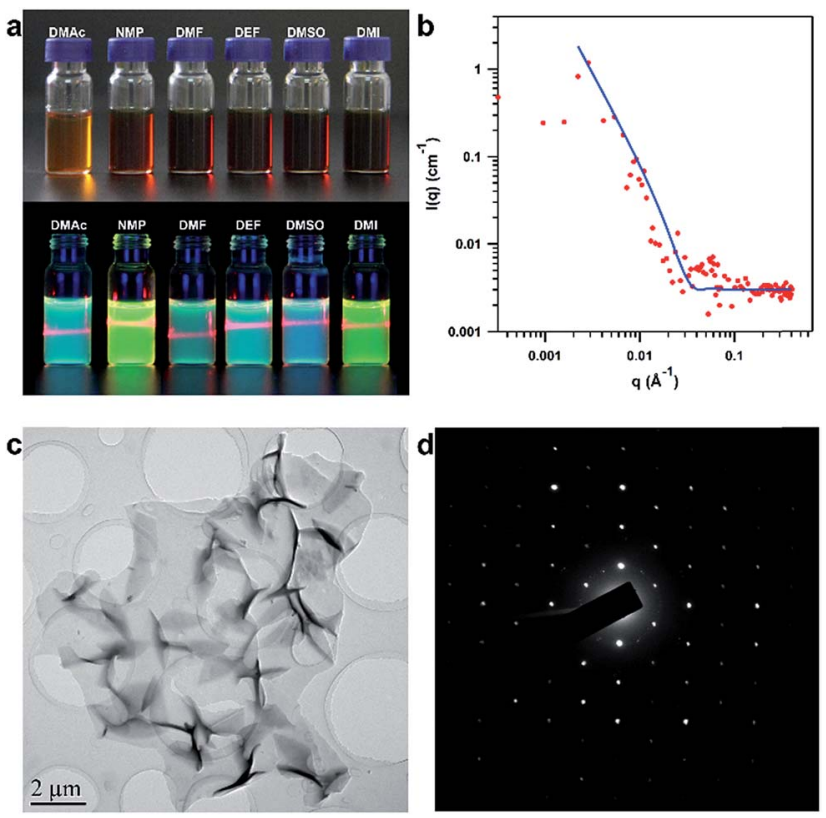

Fig. 3 (a) Photos of the PyVg-COF dissolved in various solvents. Top: concentrated solutions under sunlight; bottom: dilute solutions under $365 \mathrm{~nm}$ UV light showing the Tyndall effect. (b) SANS of a DMSO- $d_{6}$ solution containing concentrated PyVg-COF. The blue line is the curve of a typical 2D planar model. (c) TEM image of a nanosheet of the PyVg-COF at low magnification. (d) SAED of the PyVg-COF nanosheet.
DMAc, and 1,3-dimethyl-2-imidazolidinone (DMI) (Fig. 3a). Among these solvents, the PyVg-COF was most soluble in DEF $\left(8.3 \mathrm{mg} \mathrm{mL}^{-1}\right)$, and it was least soluble in DMAc $\left(2.1 \mathrm{mg} \mathrm{mL} \mathrm{m}^{-1}\right)$ (Fig. S7 and Table S5 $\dagger$ ). Such high solubility was in sharp contrast to traditional COFs which were not soluble. The concentrated PyVg-COF solutions were rather stable, and no sediment formed even when the solutions were left under ambient conditions for more than six months, in sharp contrast to traditional COF dispersions, which show low stability and a rapid settling process. To gain insight into the solubilisation mechanism, we calculated the inter-layer interactions and the interactions of the COF skeletons and the solvents (Table S6 $\dagger$ ). For PyVg-COF, the inter-layer interaction was as low as $2.22 \mathrm{eV}$, while the interactions of COF skeletons and THF and water solvents were -1.36 and $-1.95 \mathrm{eV}$, respectively. Since the solubility is the consequence of the difference value of the skeleton-solvent interactions and the inter-layer interactions, it is comprehensible that the PyVg-COF could not dissolve in these two solvents. In sharp contrast, the skeleton-solvent interactions for DMAc, NMP, DMF and DEF were as high as $-5.74,-4.86,-4.87$ and $-5.12 \mathrm{eV}$, which were much higher than the inter-layer interaction in terms of the absolute value. Thus, PyVg-COF could readily dissolve in the above solvents, while the solubility enhancement for the four solvents also matched well with the experimental trend. As a control, we also calculated the skeleton-solvent interactions and the inter-layer interaction for a neutral COF which has almost the same structure but in AA stack mode (Table S6 $\dagger$ ). In this case, the inter-layer interaction was too large compared with the skeleton-solvent interactions to allow the COF to be soluble. These results clearly revealed that the essence of the solubility was the weak inter-layer interactions and remarkable skeleton-solvent interactions.

After dissolving, the PyVg-COF could self-exfoliate into monolayers or multilayers that were well dispersed in solution and exhibited a clear Tyndall effect (Fig. 3a). Since the fluorescence of the Py monomer was quenched upon aggregation, we could determine the critical aggregation concentration (CAC) of the PyVg-COF solution by taking advantage of the concentrationdependent fluorescence quenching, which revealed that the CAC of the PyVg-COF solution was $0.03 \mathrm{mg} \mathrm{mL}^{-1}$, below and above which monolayers and multilayers were formed, respectively (Fig. S8†). To investigate the aggregation behaviour of the PyVgCOF in solution, we conducted small-angle neutron scattering (SANS) measurements of a concentrated solution of the PyVgCOF in DMSO- $d_{6}$ (Fig. 3b). By virtue of the nature of neutron scattering, a large difference of scattering length density, namely, high contrast, between deuterated solvent and nanosheets could be realised. Therefore, SANS techniques can provide more convincing scattering data for these COF materials compared to small angle X-ray scattering methods. ${ }^{35-37}$ The data were well fitted with a typical 2D planar model in which nanosheets with sizes larger than $1 \mu \mathrm{m}$ could be confirmed from data fitting in the low $Q$ range $\left(0.001\right.$ to $\left.0.02 \AA^{-1}\right) \cdot{ }^{35-37}$ The SANS data fitted in the high $Q$ range ( 0.03 to $0.4 \AA^{-1}$ ), especially considering the match of the first oscillation peak at $c a$. $0.06 \AA^{-1}$, suggested that the thicknesses of the nanosheets are approximately 10 to 
$15 \mathrm{~nm}$. These results confirm the large sizes of PyVg-COF and the formation of stacking aggregates of the monolayer nanosheets at high concentration. It can be concluded from the SANS studies that the PyVg-COF could dissolve in solvents and form true solutions, while it retained the 2D skeleton and formed aggregated structures with thickness of nanometre precision.

The structure of the PyVg-COF nanosheets was observed by high-resolution transmission electron microscopy (HR-TEM). The TEM image clearly revealed large-area nanosheets with size up to $10 \mu \mathrm{m}$ (Fig. 3c and S9†). TEM of the nanosheets also allowed the direct visualisation of the ordered rhombus polygon textures (Fig. S10†). Notably, the selected-area electron diffraction (SAED) pattern showed a highly ordered arrangement with independent diffraction points, which could only be observed in the case of highly crystalline materials, thus further confirming the high intra-layer crystallinity of the PyVg-COF (Fig. 3d). Therefore, the present PyVg-COF simultaneously possesses two distinct features, namely, crystallinity and solubility, which are highly desired for solution-processable 2D polymers. The atomic force microscopy (AFM) images revealed that the PyVg-COF nanosheets possessed smooth morphologies with heights ranging from 0.65 to $1.5 \mathrm{~nm}$ (Fig. S11†), indicative of monolayer and multilayer formations.

The high solubility of the PyVg-COF allowed us to prepare its films in a solution-processed manner. The simplest method involves drop-casting the PyVg-COF solution onto substrates, followed by thermal evaporation of the solvents. Scanning electron microscopy (SEM) images revealed continuous films with smooth morphology (Fig. S12†). Grazing incidence wide-angle X-ray scattering (GIWAXS) of the films indicated the "face-on" orientation of the COF skeletons parallel to the substrate (Fig. S13†). This facile method allows the preparation of thin films on conductive or insulating substrates and can be used for the fabrication of optoelectronic devices with various configurations (vide infra).

Upon further evaluation of the structural features of the PyVg-COF, we developed the first electrophoretic deposition (EPD) method for preparing ionic COF films by taking advantage of its highly charged skeleton (Fig. 4a). An EPD method for preparing metal-organic framework (MOF) films has been reported; ${ }^{38,39}$ however, unstable MOF suspensions that competitively form sediments must be used during the EPD process. By virtue of the high solubility and stable dispersibility of the PyVgCOF, EPD of the present COF system reproducibly provides high-quality films. We set up a two-electrode EPD system (Fig. S14 $\dagger$ ) in which various conductive substrates, such as indium tin oxide (ITO), gold and stainless steel, could serve as the working and counter electrodes. Under the optimal reaction conditions, DMI was used as the solvent, and the PyVg-COF concentration was $4.9 \mathrm{mg} \mathrm{mL}^{-1}$. EPD was conducted by applying a constant DC voltage. As the EPD proceeded, the working electrode connected to the cathode of the power supply gradually turned brown, as identified by the naked eye. After the EPD process, the PyVg-COF thin films were transparent and brown, while the thick films appeared almost black (Fig. 4b). Infrared spectra of the PyVg-COF films revealed that the structures were stable during the EPD process (Fig. S15†). SEM images of the films showed that their morphologies were
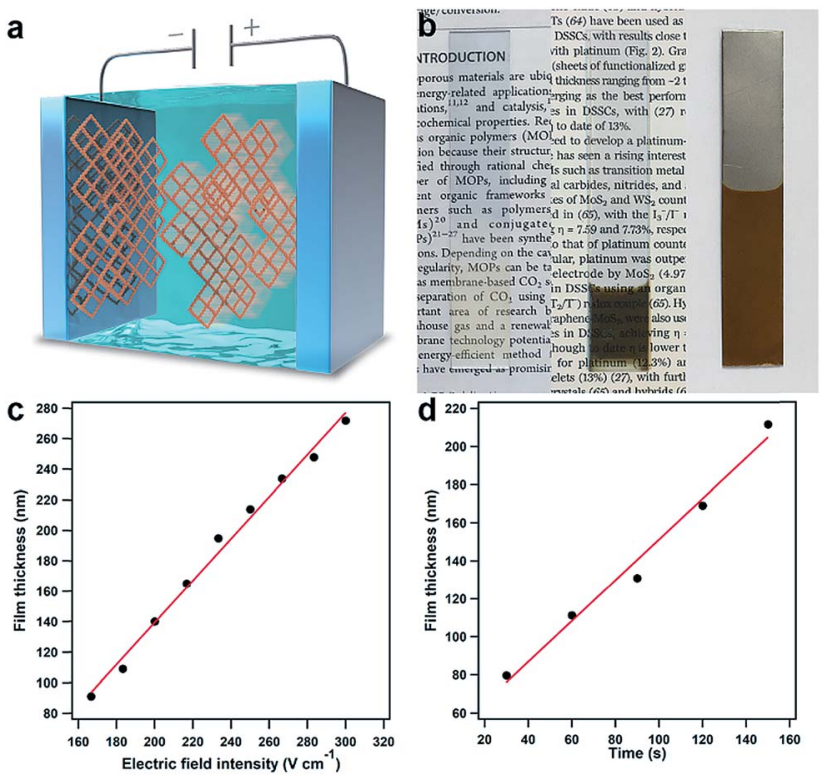

Fig. 4 (a) Scheme illustrating the principle of COF EPD film growth showing the attraction of the positively charged COF nanosheets toward the negatively charged electrode using an applied electric field. (b) Photos of the COF EPD films (left: thin film on ITO; middle: thick film on ITO; and right: thick film on stainless steel). The width of the films was $14 \mathrm{~mm}$. (c) Dependence of film thickness on the electric field intensity at an EPD time of 20 s. (d) Dependence of film thickness on the EPD time at an electric field intensity of $33.3 \mathrm{~V} \mathrm{~cm}-1$

substrate-dependent (Fig. S16 $\dagger$ ). The EPD films retained their solubility, porosity and crystallinity (Fig. S17-S19†).

A distinct feature of EPD is the ability to produce COF films with good control over their thickness by regulating the electric field intensity and the EPD time. We prepared films at various electric field intensities and EPD times and measured the film thicknesses. For instance, at an EPD time of $20 \mathrm{~s}$, the thickness of the film was linearly dependent on the electric field intensity, and the slope of these data showed that the thickness increased by $1.4 \mathrm{~nm} /\left(\mathrm{V} \mathrm{cm}^{-1}\right)$ (Fig. $\left.4 \mathrm{c}\right)$. On the other hand, the thickness of the EPD films could also be regulated by controlling the EPD time under constant electric field intensity (e.g., $33.3 \mathrm{~V} \mathrm{~cm}^{-1}$ ), and the thickness increased linearly by $1.1 \mathrm{~nm} \mathrm{~s}^{-1}$ (Fig. 4d). Notably, the developed EPD method was a rapid and highthroughput method for film preparation; for instance, the production of a $70 \mathrm{~nm}$-thick film required only $20 \mathrm{~s}$ even with a low electric field intensity $\left(33.3 \mathrm{~V} \mathrm{~cm}^{-1}\right)$ (Fig. S20†). The tunability of the film thickness determines the compatibility with various device applications. The films were only deposited on the electrodes and had the same size and shape as the employed electrodes. Thus, the present EPD method produces high-quality films, with synthetically controlled thickness, size, and shape.

The solution processability of the PyVg-COF provides an opportunity to explore some of its basic optoelectronic properties, such as electrical conductivity, which is important for COF materials, but there is lack of reliable investigations. The most common method for measuring this parameter is to use pressed pellets made by compressing COF powders. However, 
this method has large grain-boundary resistance and can be poorly reproducible. ${ }^{40}$ Furthermore, the random orientation of crystallites in pressed pellets implies that, for materials exhibiting anisotropic conduction, the observed bulk conductivity of the pellet is a weighted average of the electrical conductivity in each crystallographic direction. To measure the intra-layer (horizontal direction) and inter-layer (vertical direction) conductivity, we prepared the PyVg-COF films by drop-casting the COF solution onto ITO and glass substrates, respectively. The horizontal and vertical conductivities were measured in two types of device structures in a glovebox at $25{ }^{\circ} \mathrm{C}$ (Fig. S21 $\dagger$ ). The vertical conductivity of the COF film exhibited a linear current-voltage ( $I-$ $V)$ profile, indicative of ohmic conduction (Fig. $5 \mathrm{a}$, red curve). The slope yielded an exceptional conductivity of $0.4 \mathrm{~S} \mathrm{~m}^{-1}$, which is the highest value among the reported conductive COFs (Table $\left.\mathrm{S} 6{ }^{\dagger}\right)$. Notably, the reported conductive COFs in their assynthesised states are usually insulators, and their conductivity could be improved just by oxidation with iodine or doping with redox-active molecules, which occupy the pore space and cause the doped COFs to be nonporous (Table S6 $\dagger$ ). In comparison, the $\mathrm{PyV}$-COF films in the as-synthesised states exhibited inherently high vertical conductivity, and the pores were not occupied by oxidants or redox-active molecules; thus, the COF films described herein are more useful for further functional exploration. In sharp contrast to the vertical measurements, the horizontal measurements of the COF films showed negligible current with a conductivity of only $1.8 \times 10^{-10} \mathrm{~S} \mathrm{~m}^{-1}$ (Fig. 5a, black curve). To verify these results, we used a four-contact probe method to measure the horizontal conductivity. ${ }^{40}$ Unfortunately, the current was below the detection limit of the machine. To clarify the origin of the insulating behaviour in the horizontal direction, we utilised a two-contact probe method to measure the conductivity of several single COF nanosheets with large sizes and different thicknesses that had been prepared by drop-casting from a dilute solution (ESI Fig. S22†)..$^{40}$ Again, no current was observed even over a much wider voltage range. This result indicated that the insulating behaviour of the PyVg-COF in the horizontal direction is an intrinsic property rather than arising from grain-boundary resistance or edge defects in the nanosheets. All these results confirmed the electrically anisotropic nature of the PyVg-COF; holes or electrons could hop through the individual inter-layer donor or acceptor $\pi$-columnar arrays, while intra-layer
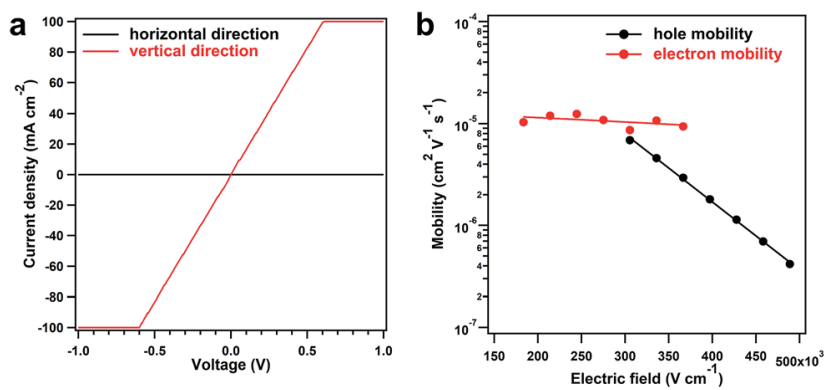

Fig. 5 (a) $J-V$ curves of the $1.6 \mu$ m-thick COF films measured in the horizontal (black line) and vertical (red line) directions. (b) Dependence of the hole (black line) and electron (red line) mobility in the COF films on the electric field in the vertical direction by a TOF method. conduction is inhibited because of charge recombination in the interlaced donor-acceptor networks. In principle, 2D COF materials should be anisotropic because they are crosslinked through covalent bonds in two dimensions and stack with each layer via $\pi-\pi$ interactions in the third dimension. However, to date, the anisotropic property has not been confirmed experimentally due to the poor processability of COF powders, although theoretical simulations have previously predicted this property. ${ }^{41}$ By virtue of its solution processability, the present COF system allows the anisotropic properties to be reliably measured and could thus serve as a model for investigating other basic behaviours of $2 \mathrm{D}$ porous materials.

To gain insight into the anisotropic conductivity, we measured the carrier mobility of the COF films. The horizontal mobility was measured by constructing a field-effect transistor in which a COF film acted as the active layer. However, no reliable current was observed, indicating that intra-layer carrier transmission was inhibited. On the other hand, the vertical mobility was measured by fabricating a sandwich-type diode in which a COF film served as the active layer via a time-of-flight (TOF) method, which is widely recognised as the most accurate method to determine the carrier mobility. ${ }^{42-44}$ Upon increasing the electric field intensity, the transient time of the holes increased significantly, while that of the electrons increased slightly (Fig. S23 and $\mathrm{S} 24 \dagger)$. Thus, both the hole and electron mobilities exhibited linear yet negative dependencies on the logarithm of the electric field intensity, and the hole mobility drastically decreased when the electric field intensity was increased (Fig. 5b). These results suggested that the built-in electric field of the COF films had negative effects on carrier migration, and hole transport was more likely to be hindered. We also calculated the intrinsic carrier mobility at zero electric field $\left(\mu_{0}\right)$, which was $9.0 \times 10^{-4}$ and $1.4 \times 10^{-5} \mathrm{~cm}^{2} \mathrm{~V}^{-1} \mathrm{~s}^{-1}$ for holes and electrons, respectively. These results revealed that the COF films presented high yet balanced carrier mobility, which was the essence of the exceptional vertical conductivity.

\section{Conclusions}

In summary, our findings represent a new strategy for the direct preparation of COF solutions and anisotropic films. The design principle of incorporating crystalline and ionic building blocks affords the resulting COFs with simultaneous crystallinity and remarkable solubility, enables solution processability of COFs into high-quality large-area films in a high-throughput and efficient manner, and allows control over the film thickness. For the first time, the anisotropic electric properties of COF materials were determined. Our conductivity results are encouraging and will inspire further structural explorations. We anticipate that solution-processable COFs and their semiconducting films will find a wide variety of applications in transistors, diodes, photocatalysis and energy storage.

\section{Conflicts of interest}

There are no conflicts to declare. 


\section{Acknowledgements}

This work was supported by the National Natural Science Foundation of China (21334002, 51521002, and 51473052), the National Basic Research Program of China (973 Program) (2014CB643504 and 2013CB834705), the Introduced Innovative R\&D Team of Guangdong (201101C0105067115), and the Thousand Youth Talents Plan. GIWAXS experiments were carried out at the State Key Lab of Luminescent Materials and Devices at South China University of Technology.

\section{Notes and references}

1 N. Huang, P. Wang and D. Jiang, Nat. Rev. Mater., 2016, 1, 16068.

2 C. S. Diercks and O. M. Yaghi, Science, 2017, 355, 923.

3 X. Feng, X. Ding and D. Jiang, Chem. Soc. Rev., 2012, 41, 6010.

4 P. J. Waller, F. Gándara and O. M. Yaghi, Acc. Chem. Res., 2015, 48, 3053.

5 H. Xu, J. Gao and D. Jiang, Nat. Chem., 2015, 7, 905.

6 L. Ascherl, T. Sick, J. T. Margraf, S. H. Lapidus, M. Calik, C. Hettstedt, K. Karaghiosoff, M. Döblinger, T. Clark, K. W. Chapman, F. Auras and T. Bein, Nat. Chem., 2016, 8, 310.

7 T. Ma, E. A. Kapustin, S. X. Yin, L. Liang, Z. Zhou, J. Niu, L.-H. Li, Y. Wang, J. Su, J. Li, X. Wang, W. D. Wang, W. Wang, J. Sun and O. M. Yaghi, Science, 2018, 361, 48.

8 H. Xu, S. Tao and D. Jiang, Nat. Mater., 2016, 15, 722.

9 E. Jin, M. Asada, Q. Xu, S. Dalapati, M. A. Addicoat, M. A. Brady, H. Xu, T. Nakamura, T. Heine, Q. Chen and D. Jiang, Science, 2017, 357, 673.

10 Z.-F. Pang, S.-Q. Xu, T.-Y. Zhou, R.-R. Liang, T.-G. Zhan and X. Zhao, J. Am. Chem. Soc., 2016, 138, 4710.

11 N. Huang, L. Zhai, D. E. Coupry, M. A. Addicoat, K. Okushita, K. Nishimura, T. Heine and D. Jiang, Nat. Commun, 2016, 7, 12325.

12 S. Wan, J. Guo, J. Kim, H. Ihee and D. Jiang, Angew. Chem., Int. Ed., 2009, 48, 5439.

13 J. W. Colson, A. R. Woll, A. Mukherjee, M. P. Levendorf, E. L. Spitler, V. B. Shields, M. G. Spencer, J. Park and W. R. Dichtel, Science, 2011, 332, 228.

14 C. S. Diercks, S. Lin, N. Kornienko, E. A. Kapustin, E. M. Nichols, C. Zhu, Y. Zhao, C. J. Chang and O. M. Yaghi, J. Am. Chem. Soc., 2018, 140, 1116.

15 T. Sick, A. G. Hufnagel, J. Kampmann, I. Kondofersky, M. Calik, J. M. Rotter, A. Evans, M. Döblinger, S. Herbert, K. Peters, D. Böhm, P. Knochel, D. D. Medina, D. Fattakhova-Rohlfing and T. Bein, J. Am. Chem. Soc., 2018, 140, 2085.

16 D. N. Bunck and W. R. Dichtel, J. Am. Chem. Soc., 2013, 135, 14952. 17 S. Wang, Q. Wang, P. Shao, Y. Han, X. Gao, L. Ma, S. Yuan, X. Ma, J. Zhou, X. Feng and B. Wang, J. Am. Chem. Soc., 2017, 139, 4258.

18 Y. Yang, F. Bu, J. Liu, I. Shakir and Y. Xu, Chem. Commun., 2017, 53, 7481.

19 S. Chandra, S. Kandambeth, B. P. Biswal, B. Lukose, S. M. Kunjir, M. Chaudhary, R. Babarao, T. Heine and R. Banerjee, J. Am. Chem. Soc., 2013, 135, 17853.
20 I. Berlanga, I. Berlanga, M. L. Ruiz-González, J. M. GonzálezCalbet, J. L. G. Fierro, R. Mas-Ballesté and F. Zamora, Small, 2011, 7, 1207.

21 H. Sahabudeen, H. Qi, B. A. Glatz, D. Tranca, R. Dong, Y. Hou, T. Zhang, C. Kuttner, T. Lehnert, G. Seifert, U. Kaiser, A. Fery, Z. Zheng and X. Feng, Nat. Commun., 2016, 7, 13461.

22 K. Dey, M. Pal, K. C. Rout, S. K. H, A. Das, R. Mukherjee, U. K. Kharul and R. Banerjee, J. Am. Chem. Soc., 2017, 139, 13083.

23 B. J. Smith, L. R. Parent, A. C. Overholts, P. A. Beaucage, R. P. Bisbey, A. D. Chavez, N. Hwang, C. Park, A. M. Evans, N. C. Gianneschi and W. R. Dichtel, ACS Cent. Sci., 2017, 3, 58. 24 A. M. Evans, L. R. Parent, N. C. Flanders, R. P. Bisbey, E. Vitaku, M. S. Kirschner, R. D. Schaller, L. X. Chen, N. C. Gianneschi and W. R. Dichtel, Science, 2018, 361, 52. 25 S. Mitra, S. Kandambeth, B. P. Biswal, M. A. Khayum, C. K. Choudhury, M. Mehta, G. Kaur, S. Banerjee, A. Prabhune, S. Verma, S. Roy, U. K. Kharul and R. Banerjee, J. Am. Chem. Soc., 2016, 138, 2823.

26 G. Das, T. Skorjanc, S. K. Sharma, F. Gándara, M. Lusi, D. S. S. Rao, S. Vimala, S. K. Prasad, J. Raya, D. S. Han, R. Jagannathan, J.-C. Olsen and A. Trabolsi, J. Am. Chem. Soc., 2017, 139, 9558.

27 N. Huang, P. Wang, M. A. Addicoat, T. Heine and D. Jiang, Angew. Chem., Int. Ed., 2017, 56, 4982.

28 H. Ma, B. Liu, B. Li, L. Zhang, Y.-G. Li, H.-Q. Tan, H.-Y. Zang and G. Zhu, J. Am. Chem. Soc., 2016, 138, 5897.

29 N. Huang, X. Chen, R. Krishna and D. Jiang, Angew. Chem., Int. Ed., 2015, 54, 2986.

30 Z. Li, X. Feng, Y. Zou, Y. Zhang, H. Xia, X. Liu and Y. Mu, Chem. Commun., 2014, 50, 13825.

31 N. Huang, R. Krishna and D. Jiang, J. Am. Chem. Soc., 2015, 137, 7079.

32 W. Lu, D. Yuan, J. Sculley, D. Zhao, R. Krishna and H.-C. Zhou, J. Am. Chem. Soc., 2011, 133, 18126.

33 Y. J. Lee, S. N. Talapaneni and A. Coskun, ACS Appl. Mater. Interfaces, 2017, 9, 30679.

34 W. Lu, J. P. Sculley, D. Yuan, R. Krishna, Z. Wei and H.-C. Zhou, Angew. Chem., Int. Ed., 2012, 51, 7480.

35 T. Li, A. J. Senesi and B. Lee, Chem. Rev., 2016, 116, 11128.

36 M. Lotya, A. Rakovich, J. F. Donegan and J. N. Coleman, Nanotechnology, 2013, 24, 265703.

37 I. W. Hamley, J. Hutchinson, S. Kirkham, V. Castelletto and A. Kaur, Langmuir, 2016, 32, 10387.

38 I. Hod, W. Bury, D. M. Karlin, P. Deria, C.-W. Kung, M. J. Katz, M. So, B. Klahr, D. Jin, Y.-W. Chung, T. W. Odom, O. K. Farha and J. T. Hupp, Adv. Mater., 2014, 26, 6295.

39 W.-J. Li, M. Tu, R. Cao and R. A. Fischer, J. Mater. Chem. A, 2016, 4, 12356.

40 L. Sun, S. S. Park, D. Sheberla and M. Dincă, J. Am. Chem. Soc., 2016, 138, 14772.

41 D. Er, L. Dong and V. B. Shenoy, J. Phys. Chem. C, 2016, 120, 174.

42 A. Kokil, K. Yang and J. Kumar, J. Polym. Sci., Part B: Polym. Phys., 2012, 50, 1130.

43 H. Bässler, Polym. Adv. Technol., 1998, 9, 402.

44 H. Bässler and A. Köhler, Top. Curr. Chem., 2012, 312, 1. 